\title{
Emprendimiento Social. Autoempleo y extracción del valor en la era post-crisis
}

\author{
Hugo Valenzuela-García'; José Luis Molina²; Miranda J. Lubbers ${ }^{3}$; Paula Escribano4; \\ Sandrine Fuentes ${ }^{5}$
}

Recibido: 7 de febrero de 2019/ Aceptado: 24 de abril de 2019

\begin{abstract}
Resumen. El fenómeno del emprendimiento social surgido en Cataluña a partir de la crisis del 2008 se presenta como una alternativa ocupacional promovida por actores financieros y administraciones públicas para responder a dos problemas agravados por la crisis financiera: la necesidad de crear empleo estable y los retos sociales y medioambientales. Este tipo de emprendimiento, liderado por una clase creativa auto-empleada, se enfrenta a las tensiones surgidas de equilibrar las aspiraciones personales y convicciones sociales contra las presiones del mercado. Sostenemos que la nueva etiqueta -emprendimiento social- es parte de una política que refleja la alianza de intereses financieros, escuelas de negocios y el estado neoliberal orientada a cubrir la fractura material e ideológica dejada por la crisis económica. Este texto aporta, por una parte, una contribución empírica al universo del emprendimiento social (basada en el análisis de 43 estudios de caso de empresas sociales, 93 encuestas de emprendedores y una base de datos de 347 entidades) y, por la otra, una aproximación teórica para entender esta expansión capitalista y su creciente sofisticación ideológica y práctica en los procesos de extracción del valor.
\end{abstract}

Palabras clave: Emprendimiento social; valor; restructuración laboral; externalización; Estado neoliberal.

\section{[en] Social Entrepreneurship. Self-employment and value extraction in the post-crisis era}

\begin{abstract}
Social Entrepreneurship emerged in Catalonia after 2008 and offered an occupational alternative fostered by financial actors and public administrations to respond to two major problems aggravated by the financial crisis: the creation of stable work and the resolution of socio-ecological problems. The new entrepreneurship, headed by a self-employed creative class, faces the tensions of balancing market orientation and social objectives. We hold sustain that the new label - social enterprise - We argue that the new label - social entrepreneurship - is part of a policy that reflects the alliance between financial interests, business schools and the neoliberal state aimed at covering the material and ideological fracture left by the economic crisis.. The paper provides, on the one hand, an empirical contribution to the universe of social entrepreneurship (based on the analysis of 43 case studies of social enterprises, 93 surveys of entrepreneurs and a database of 347 entities) and, on the other, a theoretical approach to better understand this evolving phenomenon as the product of new and sophisticated ways of Capitalist value extraction.
\end{abstract}

Keywords: Social entrepreneurship; value; work restructuring; outsourcing; neoliberal state.

\footnotetext{
Universidad Autónoma de Barcelona, hugo.valenzuela@uab.cat

Universidad Autónoma de Barcelona, joseluis.molina@uab.es

Universidad Autónoma de Barcelona, mirandajessica.lubbers@uab.cat

Universidad Autónoma de Barcelona, paula.escribano@uab.cat

Universidad Autónoma de Barcelona, sandrine.fuentes@uab.cat
} 
Sumario. 1. Introducción. 2. Génesis del emprendimiento social. 3. El emprendedor social y la "Clase Creativa". 4. Metodología, 5. El emprendedor social en Cataluña. 6. Conclusiones. 7. Referencias bibliográficas.

Cómo citar: Valenzuela-García, H.; Molina, J. L.; J. Lubbers, M.; Escribano, P; Fuentes, S. (2019). Emprendimiento Social. Autoempleo y extracción del valor en la era post-crisis, en Revista de Antropología Social 28(2), 371-390.

\section{Introducción}

A social enterprise is actually a failed business (...) once it becomes profitable, it's just a good enterprise (Eyal Halamish, co-fundador de OurSay, en Waters, 2017)

Lucía es una joven arquitecta de 37 años, entusiasta y vital. Hasta 2007 gozaba de un contrato estable en un estudio de diseño de vivienda de protección oficial. Trabajaba mucho y duro, aunque disfrutaba de un nivel de vida elevado. Con la crisis económica la facturación de la empresa empieza a resentirse. Se reducen drásticamente sus contratos y deja de percibir subvenciones públicas, su principal fuente de ingreso. Como consecuencia, la empresa realiza una reestructuración laboral: despide a algunos empleados, y a otros, como a Lucía, les proponen establecerse como trabajadores autónomos pero les reducen su contrato laboral a la mitad. Son tiempos difíciles: su padre enferma, se divorcia de su marido, se queda a cargo de su hija de cuatro años $y$, con la mitad de ingresos, empieza a tener dificultades para cubrir los gastos domésticos, las facturas y la hipoteca.

Lucía siempre tuvo cierta sensibilidad social. Le interesaban no solo el diseño de las viviendas sino también las necesidades y las condiciones de vida de los moradores, por lo general personas humildes que no podían costearse una vivienda de mercado. Dada esa inquietud, y el hecho de que su salario se redujese a la mitad, decidió arriesgarse:

...tenía ganas de salir de aquella empresa (...) estaba en un momento de cambio personal total. Sentí que no estaba totalmente realizada en mi vertiente social y decidí embarcarme en eso, pero inicialmente no me planteaba que fuera a suponer más del cincuenta por ciento de mi sueldo.

En 2011 crea una empresa online (sic) "para mejorar el día a día de las mujeres", ofreciendo productos femeninos asequibles, "al alcance de todas" y "naturales": ropa íntima, productos de higiene femenina, copas menstruales, etc. Se asesora en una consultora y se constituye en cooperativa, la forma jurídica más conveniente, asociándose con su excuñada (diseñadora gráfica que vive en Rotterdam), una amiga arquitecta que exporta los productos desde Guinea, y una tercera socia experta en lenguaje y computación que gestiona los pedidos y la página web.

En un inicio recurre a sus ahorros y pide ayuda a su pequeña red de familiares y amigos. Su antigua red laboral le proporciona el capital social básico (contactos y consumidores) y sus estudios le otorgan el capital humano y cultural necesarios para 
moverse con solvencia en el nuevo contexto empresarial. Además, gana dos concursos de iniciativas empresariales patrocinados por fundaciones bancarias que le dotan de asesoría, promoción y formación.

El trayecto no ha sido sencillo. Como reconoce ella misma, "nadie podía imaginar esta carrera de obstáculos cuando acabé la licenciatura". Solo desde hace unos meses ha llegado a obtener beneficios regulares, pero durante muchos años ha formado parte del amplio sector joven y mileurista.

Al principio gestionaba su empresa desde la biblioteca municipal de su barrio, lo cual le permitía separar la actividad económica y la frenética tarea doméstica en solitario -cuidar de su hija, llevarla a la escuela y a actividades extraescolares, mantener el hogar, limpiar y cocinar, etc. Al tiempo, decide alquilar una "oficina" por horas, consistente en una pequeña mesa y conexión a Internet en un espacio de co-working en una céntrica calle de Barcelona. Comparte el espacio con decenas de personas como ella -jóvenes vitales, creativos y con interesantes proyectosque, además de procurarse el sustento, tratan de cambiar un poco el mundo. Pero, paradójicamente, sus atareadas vidas les dejan poco espacio para interaccionar e intercambiar ideas y proyectos en esos espacios donde la colaboración es en realidad escasa. El caso de Lucía es representativo del nuevo emprendedor social en Cataluña, que a su vez muestra similitudes con casos hallados en el resto de España, Europa (Doherty, Haugh y Lyon, 2014), Suráfrica (Littlewood y Holt, 2018) o Asia (Moon y Koh, 2015).

En este artículo trataremos de mostrar que el emprendimiento social obedece a un fenómeno emergente y estructural; una manifestación de una realineación del capitalismo que afecta al trabajo en el siglo XXI (autoempleo, emprendimiento o do-it-yourself) y a la manera en que se crea, extrae y explota el valor social colectivo. Esta transformación global está liderada por una "clase creativa" auto-empleada promocionada por actores financieros clave que han contado con la connivencia del estado neoliberal (véase Gill y Pratt, 2008: 29; Wacquant, 2009; Hilgers, 2012). Para ello abordaremos, en primer lugar, la génesis de este fenómeno en España para pasar, a continuación, a describir las diversas conceptualizaciones teóricas existentes. Una vez descrita la metodología empleada pasaremos a describir los resultados alcanzados y a valorar su alcance.

\section{Génesis del emprendimiento social}

A pesar de que la economía social y solidaria posee una larguísima y rica tradición en Cataluña y otras regiones de España (cooperativas, empresas de inserción, ONGs, etc.), el así llamado emprendimiento social es un sector relativamente novedoso. El concepto lo lanza la red internacional Ashoka a partir de los años 80 (Bornstein \& Davis, 2010) y en España solo se populariza realmente a partir de 2010 y en un contexto revelador.

En España, como en muchos otros países europeos "periféricos" (Chipre, Grecia, Irlanda, Portugal, Italia, etc.), la principal respuesta a la dura crisis económica de 2008, al contrario que en otros lugares y periodos (por ejemplo, el keynesianismo tras la Gran Depresión norteamericana), tomó la forma de una severa política de recortes y de reducción de deuda pública que tuvo efectos devastadores en el trabajo, el consumo privado y la inversión pública: entre 2010 y 2014 el gobierno español, 
presionado por Europa, subió impuestos, congeló pensiones, redujo el salario de funcionarios un 5\%, el presupuesto de sanidad un 10\%, un 19\% el de educación (un $68 \%$ el de educación compensatoria), un 30\% el destinado a $\mathrm{I}+\mathrm{D}+\mathrm{I}$ y un $13 \%$ el de ayudas sociales (Del Pino, 2013; Conde-Ruíz, Díaz, Marín et al., 2016). Nada de esto se ha recuperado a día de hoy, una década después. España, después de Chipre, encabezó el ranking europeo de desigualdad. El trabajo no solo decreció en cantidad sino también en calidad, a través de toda una cascada de medidas (en nombre de la competitividad) para abaratar despidos, reducir salarios y recortar derechos laborales que redundaron en la expansión del trabajo temporal, precarizado, flexible e informal -que alcanzó un 24,6\% del PIB en 2014, frente al 17,8\% anterior al 2008 (el doble que en Reino Unido, Francia y Alemania) (Mount, 2014). La destrucción de empleo se hizo particularmente patente en la pequeña y mediana empresa, que en España supone el 99,8\% del tejido empresarial.

Para preservar el sistema financiero, sobre el cual se cimienta toda la estructura económica del crédito (Martín Belmonte, 2011), el gobierno destinó buena parte de los recursos prestados por la Unión Europea a socorrer a la banca y a comprar deuda soberana: en cinco años (de 2009 a 2014) los bancos españoles (Bankia, Banco de Valencia, CAM, Novacaixagalicia, Catalunya Banc, etc.) reciben nada menos que 77.000 millones de euros. Además, la restructuración del sistema financiero implicó la eliminación de las Cajas de Ahorro, instituciones sin ánimo de lucro que en España han tenido históricamente una función asistencial muy significativa. En 2008 aportaban más de 2 billones de euros al gasto social, pero en 2014 prácticamente desaparecen (Montero, Arcenegui, Martín Lozano et al., 2015).

A raíz de la crisis surge, en distintos puntos de Europa, un amplio movimiento cívico que reclama la necesidad de supeditar el beneficio económico al interés social (Hart, 2015). Se trata de prácticas heterogéneas, producto tanto de la necesidad (aprovisionamiento económico por vías alternativas) como del desencanto por los excesos de la economía de mercado (desigualdad, contaminación medioambiental, etc.). Esta preocupación, que se remonta nada menos a los escritos de Aristóteles, resuena en conceptos más contemporáneos como economía moral (Thompson, 1971; Scott, 1976; Polanyi, 1977; Booth, 1993; Zak, 2008), democrática (Mendell, 2005), humana (Hart, Laville y Cattani, 2010) o ética (Koslowski, 2008).

En este sentido, en Cataluña se crean gran cantidad de bancos del tiempo (comunidades de intercambio de servicios donde la unidad de valor e intercambio es el tiempo de trabajo o servicio prestado), redes de trueque local (intercambios de bienes y servicios), pequeñas cooperativas de consumo ecológico (sostenible, solidario, de proximidad), mercados de autoproducción y monedas sociales. Junto a estos LETS (Local Exchange Trading Systems) (Linton, 1994; Lang, 1994), también reemergen una amplia diversidad de cooperativas, ONGs y empresas sin ánimo de lucro bajo distintas fórmulas jurídicas (SA, SL, fundaciones, etc.).

A pesar del carácter contracultural y crítico de muchas de estas propuestas, este movimiento representó para el Estado y los principales actores financieros una oportunidad para liderar el lanzamiento de una selección de iniciativas (posiblemente la más rentable), bajo el paraguas de emprendimiento social. Esta flamante etiqueta, más amable que la de empresa comercial, tiene además un seductor valor añadido (social, ecológico o solidario) y un innegable potencial tanto para rentabilizar el bien común (cooperativismo, colaboración, altruismo, etc.) como para suplir las carencias de un estado del bienestar en retirada. 
De modo general, y a pesar de las contradicciones evidentes (Valenzuela y Fuentes, 2019), una empresa social es "aquella iniciativa que cumple un misión social o medioambiental orientada a fines de mercado, utilizando soluciones innovadoras y obteniendo elevado impacto" (Dacin, Dacin, y Matear, 2010). El emprendimiento social se presenta, de este modo, como "el adalid de una verdadera revolución en el seno del capitalismo, que superará al anticuado estado del bienestar" (Mason, 2015) "y contribuirá, mediante su portentosa economía colaborativa (sharing economy), a la creación de un doble valor: económico y social" (Porter y Kramer, 2011: 64; en Driver, 2012: 421). Esta nueva oportunidad la lideran las escuelas de negocios de referencia (Harvard Business School, ESADE, etc.) que ahora tienden a introducir lo social en el diseño de sus programas y a reclutar a investigadores sociales, consultores y CEOS para trasladar, al fin y al cabo, su savoir faire comercial al campo del emprendimiento social (Austin, Stevenson y Wei-Skillern, 2006; Meyskens, Calleen, Stamp et al., 2010).

A partir de 2010 surgen varios programas de promoción del emprendimiento social, tales como Momentum (BBV y ESADE) o el Programa d'Emprendoria Social de la Caixa (en colaboración con IESE Business School), que ofrecen financiación, asesoría y oportunidades crediticias a emprendimientos sociales prometedores. Se crea de este modo un campo institucional de actividad empresarial y autoempleo que se ampara en los valores de la economía social y solidaria y ocupa el espacio del tradicional Tercer Sector (cooperativas, ONGs) (Moulaert y Ailenei, 2005).

La recepción política no es menos entusiasta. El emprendimiento social rápidamente es objeto de sustanciales incentivos privados (fundaciones bancarias), públicos (administraciones públicas) y adquiere mayor centralidad en leyes y regulaciones que enaltecen su potencial para ahorrar costes, crear empleo y estabilizarlo (Ruiz-Herrero, 2015: 16). En 2011 la UE aprueba e incluye en su agenda la Social Business Initiative (EURICSE, 2013). En 2013 se subraya lo social en la Estrategia de Emprendimiento y Empleo Joven (Ministerio de Empleo), se aprueba la ley de emprendedores y se ponen en marcha programas para potenciarlo desde la educación primaria (en Ruiz-Herrero, 2015). En 2011, la Generalitat de Catalunya lanza el programa@EmprenSocial para fomentar el emprendimiento social mediante reuniones, directorio de recursos o comunicación en los medios.

El gran potencial del emprendimiento social para transformar los problemas sociales y medioambientales en activos económicos rentables a mínimo coste público, explica que este se haya convertido en un Campo de Acción Estratégico (SAF, Strategic Action Field) no exento de conflicto de intereses por los distintos actores en liza (Molina, Lubbers, Valenzuela et al., 2018). Por una parte, hallamos al Estado, que promueve el sector porque permite crear empleo y externalizar o ahorrar el coste de muchas de sus funciones (atención social, subvenciones al tercer sector, inserción de personas en situación de vulnerabilidad, acometer problemas ecológicos, etc.). Por otra parte, tenemos a los principales actores financieros y sus fundaciones, interesados en liderar ese nuevo sector porque resulta rentable y posibilita regenerar la imagen maltrecha de la banca. Y, finalmente, hallamos a los colectivos que practican una economía alternativa, entre los que se cuentan desde los individuos solidarios convencidos (críticos del mercado), hasta los emprendedores al uso que han detectado un nuevo nicho de oportunidad, o los que se definen como emprendedores sociales, que comparten los rasgos propios de la clase creativa abandonada a su propia suerte económica. 
En definitiva, el emprendimiento social surge en medio de una tormenta perfecta: una crisis económica que exacerba el desempleo y la precariedad; la retirada del Estado del bienestar y la dejación de sus funciones, el avance de los poderes financieros globales y la existencia de un colectivo sensibilizado y altamente formado, aunque desplazado del mundo laboral. De acuerdo con una consultora entrevistada, "toda la gente que conozco en el mundo del empresariado social viene bien de la cooperación, bien del mundo asistencial".

\section{El emprendedor social y la "Clase Creativa"}

Consideramos que el emprendimiento social es el resultado de la convergencia de al menos dos procesos del desarrollo capitalista tardío: la industria creativa derivada del capitalismo cognitivo y la corriente del capital humano, de una parte. Y de la otra, el emprendimiento como forma de macro y micro-gestión expansiva que impregna prácticamente todas las esferas de la vida social. Estos procesos, sostenemos aquí, cristalizan en la figura del emprendedor social, una suerte de clase creativa auto-empleada.

El capitalismo cognitivo surge en la década de 1960 y 1970 (Drucker, 1968 1968). A diferencia del capitalismo industrial anterior -fordista y sustentado en la producción de bienes de consumo-, este se caracteriza por la producción de intangibles (servicios, diseños, prototipos o ideas), el uso intensivo de tecnologías de la comunicación y la información, y una fuerza laboral cualificada (Overell, 2008: 28; Mason, 2015). La corriente del capital humano, por otra parte, enfatiza las habilidades empáticas y emocionales (sociales) para generar valor económico (Scace, 2002: 86-87). No es por ello extraño que Michel Foucault (2008) identificase en la corriente del capital humano un paso decisivo en la colonización neoliberal de todas las esferas del sujeto, al incursionar en la personalidad, los sentimientos y las emociones del individuo.

Estas corrientes sitúan al trabajador creativo en el centro del proceso productivo, puesto que de este depende la ideación y creación del valor económico. Para promover que los trabajadores aporten sus creaciones, las formas de gestión clásicas (vigilancia, control, incentivos o prohibiciones) son sustituidas por una gestión horizontal, ambientes amables e incentivos motivacionales y post-materialistas (Inglehart, 1977, 2009); es decir, estímulos que van más allá del salario, como pueden ser el reconocimiento, la realización personal, la autonomía o la expresión de la subjetividad. "La propiedad intelectual", afirma Ross, es "el lucrativo premio del esfuerzo creativo, (...) el petróleo del siglo XXI y, por ello, gobiernos de todo el mundo han adoptado la industria creativa como modelo de plan de desarrollo regional" (en Lovink 2007: 32). En este contexto, el trabajo adquiere una dimensión significativa raramente presente en la historia (Overell, 2008: 31), pasa a ser un rasgo consustancial a la identidad del individuo (Fleming, 2014: 29). La vocación, por una parte, y la ubicuidad de las tecnologías de la información y las comunicaciones, por la otra, potencian la flexibilidad y contribuyen a lo que los teóricos autonomistas italianos denominan la fábrica social: una absorción total de la vida (el ocio, la familia, las relaciones sociales, emocionales y afectivas) por el trabajo (Hardt y Negri 2000: 291). Naturalmente, se ha señalado repetidamente, las contrapartidas de este carácter vocacional, significativo y absorbente del trabajo son la "auto-explotación", la fatiga, la incerteza y el estrés producido por la necesidad de actualizar constantemente 
conocimientos y habilidades en campos cambiantes (Kunda, 1992; Sennet 1992; Rose, 1999; De Witte 1999; Sennet, 2000; Terranova, 2000; Benner, 2002: 203; Scace, 2002: 35; Ross, 2003; Hunt, 2006; Kalleberg 2009: 9; Prentice, 2015: 107).

En el modelo de finales del S. XX y principios del S. XXI, Florida $(2010)^{6}$ definió a estos trabajadores de la industria cognitiva como la clase creativa: un sector definido por las tres $\mathrm{T}$ (talante, tolerancia y tecnología), rupturista con los espacios, normas y atuendos laborales tradicionales; flexible; joven, cualificado, cosmopolita, "conectado" e individualizado en el sentido de Beck, Boons y Lau (2003) -es decir, crítico con los patrones sociales tradicionales y sin excesivas cargas sociales-. Actualmente este sector supone el 30\% de la población activa en Estados Unidos (Florida, 2010) y Reino Unido (Clifton, 2008) y el 10\% en España (Ruíz Herrero, 2015), e incluye a tecnólogos, científicos, ingenieros, profesores, arquitectos, trabajadores del sector de la salud, consultores o artistas.

Lo que nosotros encontramos en el caso del actual empresariado social catalán es, sin embargo, una suerte de clase creativa (Florida, 2010) desprovista del amparo institucional (la empresa) y abocada, por tanto, o bien a formar parte de la periferia creativa compuesta por $m c j o b s$ y trabajos retribuidos en forma de voluntariado, becarios o aprendices (Dilly, 2006: 307); o bien a embarcarse en el emprendimiento como alternativa de auto-empleo, un "modelo neoliberal regido por los valores del espíritu empresarial, la individualización y la dependencia del patrocinio comercial" (McRobbie, 2010: 516). En efecto, el emprendimiento implica la consagración de la ideología y la praxis hegemónica de micro y macro-gestión, crecientemente proyectada sobre el resto de las esferas de la vida. Michel Foucault conceptualizó esto en la idea de biopoder: "generalizar la forma empresarial dentro del cuerpo social o tejido social (...) para convertir la vida individual misma en una especie de empresa permanente y múltiple" (2008: 241-241). Esta proyección se observa hoy en la manera de gestionar la administración pública (Ruiz-Herrero, 2015: 19), el Estado (Gill y Pratt, 2008: 29; Wacquant, 2009), las corporaciones ${ }^{7}$ y las relaciones sociales, encapsulando los principios de la economía neoclásica (individualismo, oportunismo, maximización, competitividad, etc.), que adquieren un papel central en la forma de ganarse la vida (Freeman, 2007) y expresar la subjetividad (Sennet, 1992: 219). No es por ello extraño que el colectivo de emprendedores sociales catalanes comparta muchos rasgos con la muestra del Global Entrepreneurship Monitor (Molina, Lubbers, Valenzuela et al., 2018) y con los trabajadores de la Nueva Economía (Valenzuela, Reygadas y Cruces, 2015). La diferencia en el caso del emprendimiento social estriba en que, al locus empresarial se suma la invocación a valores morales, sociales y medioambientales socialmente apreciados. ¿Qué puede haber más ético y loable que generar, en el actual contexto socioeconómico, valor económico de manera individual en base a un trabajo digno, oportunidades de inserción laboral de colectivos marginales y producción de bienes y servicios respetuosos con el medio ambiente?

El emprendimiento social en suma sería la cristalización de esos procesos, encarnados en un individuo que, frente a las adversidades, halla soluciones sostenibles y rentables independientemente del Estado, simbolizando así las aspiraciones de la

6 Omitiremos aquí, puesto que no es nuestro objetivo ni tenemos espacio, las críticas al concepto en sí mismo, que son abundantes.

7 Empresas como Google hace tiempo que incentivan las actividades emprendedoras, cediendo una porción de la jornada laboral a sus trabajadores para que estos se dediquen a sus 'propios' proyectos creativos e innovadores. 
clase media (reputación, prestigio y respetabilidad) (Freeman, 2007). Este individuo internaliza procesos de auto-gestión, auto-explotación y responsabilidad que se perciben como deseables porque se amparan en bases emocionales importantes como la auto-realización, el compromiso social, la sensibilidad medioambiental o la solidaridad (Rose, 1999; Ruiz-Herrera, 2015; Prentice, 2015: 140-200).

\section{Metodología}

Este artículo se basa en el proyecto de investigación ENCLAVE ${ }^{8}$ desarrollado en Cataluña entre 2013 y 2016. La investigación adoptó un diseño de métodos mixtos consistente en triangular datos cuantitativos y cualitativos que generó tres conjuntos de datos: primero, 43 estudios de caso en profundidad de directores, responsables o socios de empresas sociales y/o medioambientales basados en entrevistas semiestructuradas en profundidad, entrevistas estructuradas, observación participante y contactos informales a lo largo del proyecto. La entrevista comprendía una primera parte destinada a recolectar datos sobre la iniciativa (año de constitución, sector en el que opera, forma de propiedad, número de socios, empleados y voluntarios; ingresos anuales, origen del capital inicial, etc.) y una segunda parte orientada a recabar datos sobre el emprendedor (sexo, edad, nivel de estudios, empleo anterior, tipo de motivación para iniciar el negocio o expectativas de futuro a 5 años vista). Posteriormente se analizó el tipo de apoyo recibido durante la fase de lanzamiento y formación de la empresa, aplicando análisis de redes personales con la ayuda de EgoNet. Mediante el análisis de redes personales se obtiene información detallada sobre las personas que han apoyado la iniciativa y al emprendedor/a, las características sociológicas de este/a (sexo, edad, formación educativa, relación con ego, etc.), el tipo de apoyo (recursos, información, servicios, etc.), su temporalidad y direccionalidad (por ejemplo, si se da reciprocidad) y la relación entre los diferentes individuos que aparecen en la red social del entrevistado/a. En segundo lugar, se diseñó una encuesta online (disponible en tinyurl.com $/ \mathrm{h} 2 \mathrm{zxk} 46$ ) con preguntas similares (pero excluyendo las referentes a la red de apoyo), utilizando nuestra propia muestra de entrevistados y listas de difusión de asociaciones, obteniendo 93 cuestionarios válidos respondidos por gestores, socios fundadores o propietarios de las empresas y entidades. En tercer lugar, se creó una base de datos que logró obtener información sobre 347 iniciativas sociales y medioambientales en Cataluña, procedentes de tres fuentes: nuestros propios datos recabados durante la investigación, un barrido de empresas sociales que disponían de portales en Internet (mediante búsquedas con palabras clave: empresas + sociales + Catalunya, por ejemplo) y las bases de datos de las redes o asociaciones de emprendimiento social y economía solidaria (por ejemplo, el Mapa de l'Economia Solidària, la Xarxa d'Economia Solidària o el Mapa d'Actors de l'Empresa Social a Catalunya). De estas iniciativas, hemos registrado su forma legal, el sector económico y la ubicación geográfica. La recolección de datos de las empresas sociales comportó un doble control (telefónico y por correo electrónico), para asegurarnos de que seguían operativas. Consideramos que este mapa (o instantánea) es un reflejo fidedigno de este sector en Cataluña en el momento en que se realizó la investigación.

8 Social entrepreneurship: local embeddedness, social networking sites and theoretical development. MINECO (CSO2012-32635; 2013-2016). Investigador principal: José Luis Molina. Egola-GRAFO. Departament d'Antropologia Social i Cultural. Universitat Autònoma de Barcelona. 
Además, durante cinco años los miembros de equipo han creado contactos en ese sector y han realizado observación en distintos contextos y escenarios de la economía social catalana desde 2010: en empresas; en incubadoras de negocios, hubs y espacios de co-working; en ferias y actos de promoción del emprendimiento; y en cursos de formación dirigidos a empresarios. Estas observaciones han aportado datos cualitativos relativos a la idiosincrasia, hábitos e ideología de los emprendedores, interacciones informales, discursos y prácticas de los promotores, etc., que sirvieron para triangular los datos de encuestas y entrevistas. Finalmente, los miembros del equipo participaron en actividades de transferencia (charlas, talleres, seminarios...) en colaboración con los agentes y asociaciones del sector y lograron realizar entrevistas formales e informales con más de una decena de representantes públicos, consultores y expertos. En todo momento las entrevistas se efectuaron con un consentimiento informado previo por parte de los participantes y en contrapartida se les retornó un documento con las principales características de su red social. Los datos que aparecen en este texto se han anonimizado?

\section{El emprendedor social en Cataluña}

De acuerdo con nuestra muestra, el $60 \%$ de los empresarios sociales son varones, con una edad media de 42 años y un nivel formativo elevado: un $80 \%$ contaba con estudios superiores (grados y licenciaturas universitarias) y, de estos, aproximadamente un $20 \%$ disponía de postgrados (máster y doctorado) en formaciones técnicas, humanísticas o sociales. Antes de embarcarse en la empresa, el $47 \%$ trabajaba para terceros (el $28,6 \%$ era asalariado y el $18 \%$ formaba parte del grupo de dirección o gestión de empresas), el 14,3\% era autónomo y el 10\% desempleado o estudiante. Los emprendedores manifiestan, en la creación de sus empresas, que su principal motivación es social y ecológica (48.9\%), seguida de la realización personal (31.1\%) y, solo en tercer lugar, económica. La mayoría empleó servicios de asesoría empresarial profesional, considerada 'fundamental' en el $38.6 \%$ y 'bastante importante' en el $35.1 \%$ de los casos.

Tabla 1. Datos estadísticos generales del emprendedor social catalán (fuente propia)

\begin{tabular}{|l|c|c|}
\hline \multicolumn{1}{|c|}{ Atributos individuales } & Entrevistas & Encuesta online \\
\hline Mujeres & $40 \%$ & $37 \%$ \\
\hline Edad (media) & 40.23 & 42.89 \\
\hline Porcentaje de licenciados universitario & $86 \%$ & $76 \%$ \\
\hline Motivación social y ambiental & Media de $4.64 / 5$ & $48.9 \%$ \\
\hline Motivación desarrollo personal & Media de $4.70 / 5$ & $28(311 \%)$ \\
\hline Motivación económica & Media de $2.06 / 5$ & $11(12.2 \%)$ \\
\hline Ocupación previa: parado, auto-empleado, ocupación precaria. & $40 \%$ & $51 \%$ \\
\hline
\end{tabular}

9 Salvo en los casos de las cooperativas más populares, cuyo discurso y desarrollo socioeconómico es bien conocido, el resto de iniciativas y nombres de informantes han sido anonimizados y se han empleado pseudónimos en lugar de los nombres auténticos. 
Pero ¿qué es en realidad, o cómo se define, una empresa social? Si analizamos la selección de empresas sociales impulsadas por el programa del BBVA Momentum Project, en su edición 2014, hallamos cuatro iniciativas industriales: una ingeniería que diseña una aplicación móvil para mejorar el transporte público; dos iniciativas productivas (una dedicada a las pantallas y otra al vidrio) y una empresa de reciclado de residuos. También hallamos tres iniciativas de inserción laboral de personas en riesgo de exclusión y dos iniciativas para mejorar la salud y la calidad de vida de personas enfermas. Finalmente, encontramos una empresa comercial, ShopCop, cuyo objetivo es revalorizar el trabajo de diseñadores y marcas de ropa de producción local empleando a mujeres que provienen del mundo de la prostitución. Esta empresa fue objeto de una activa campaña de márquetin, recibió avales del Ministerio y la Generalitat y sus creadoras recibieron formación y coaching a cargo de ESADE.

Respecto a las iniciativas de nuestra muestra, el 60\% surge a partir del año 2008 y, según datos macro-estadísticos, significa el 7\% del tejido laboral de nueva creación. Salvo en el caso de las cooperativas, prácticamente todas las empresas sociales son pequeñas y medianas empresas urbanas y periurbanas co-gestionadas por menos de tres propietarios y con una facturación discreta (media de 100.000€/anuales) derivada en el $80 \%$ de actividades comerciales. Es destacable el hecho de que se trate de un sector altamente subvencionado: el $48 \%$ de las iniciativas ha contado con algún tipo de subsidio privado o público; donaciones (20\%), micro-financiación $(10,5 \%)$ o inversiones externas $(17,4 \%)$, mientras que solo un $9,6 \%$ ha optado a créditos bancarios. Con una media de 14 trabajadores contratados también cabe subrayar la elevada presencia de voluntarios, aprendices y personal en prácticas. La forma legal más común es la cooperativa $(56,5 \%)$, con mayores exenciones fiscales, seguida de la SL (19\%). Los encuestados eran capaces de mencionar, como media, otras 23 iniciativas sociales o ambientales dentro de su red, por tanto, es destacable el nivel de integración (embeddedness) en redes de emprendimiento social más amplias.

El principal sector de actividad económica es el de los servicios, con un $63,4 \%{ }^{10}$, destacando los destinados al cuidado (salud, educación, formación, etc.), servicios profesionales (desde la comunicación, pasando por la mensajería o el interiorismo) $\mathrm{y}$, sobre todo, la consultoría (asesoría, formación y promoción del emprendimiento social). El segundo sector en relevancia es la producción y distribución agroecológica y energética (16,1\%). Finalmente hallamos comercio $(5 \%)$, banca ética $(2 \%)$ y distintas cooperativas y centros especiales de inserción laboral. Por tanto, podemos identificar básicamente tres sectores bajo la etiqueta de empresa social, operando a diferente escala y régimen legal: el sector agroecológico y energético; un sector de iniciativas orientadas a la venta y servicios comerciales; y la consultoría. Veamos sus características y problemáticas.

El sector agroecológico y energético comprende un amplio nicho de iniciativas de tamaño variable. Primero, encontramos grandes cooperativas (como productoras de arroz del Delta del Ebro) y asociaciones clásicas de consumidores de productos ecológicos (algunas con centenares de familias asociadas y facturaciones considerables), así como firmas del ámbito de la ingeniería y la energética que apuestan por la

10 Si de la muestra eliminamos los Centros Especializados de Trabajo y las Empresas de Inserción Laboral, obtenemos un escenario más realista del emprendimiento social en Catalunya, con dos claros sectores: los servicios (jardinería, limpieza, etc.) (cerca del 64\%) y la producción, distribución y consumo de productos agroecológicos (cerca del 15\%). 
tecnología y la innovación (EnergyAll o Foodchain, por ejemplo). Estas iniciativas forman parte del tradicional tejido cooperativista catalán (Reventós, 1960) que ha sido, históricamente, una alternativa habitual para responder al desempleo estructural (Díaz, 2000).

En segundo lugar hallamos cooperativas como La Falguera, El Trífol o La Parra cuyo éxito estriba, entre otras cosas, en vincular la inserción laboral (de personas con discapacidad o en riesgo de exclusión) y la creación de un producto con alto valor añadido, a menudo ecológico (de proximidad, sostenible, tradicional, etc.). Estas últimas se consideran el paradigma y referente del sector del emprendimiento social.

En tercer lugar, hallamos un amplio conjunto de iniciativas de reciente creación orientadas a la producción y distribución a pequeña escala de productos agroecológicos como hortalizas, verduras, frutas, cereales, harinas o pan, de reciente creación y a pequeña escala. Se trata de alternativas de auto-empleo constituidas como cooperativas (porque resulta más asequible), lideradas por jóvenes de entre 30 y 45 años (en muchos casos amigos o parejas) y, en general, con formación universitaria (ciencias agrónomas, ecología, biología, arte, geografía, etc.). Sus instalaciones (molinos, cámaras, hornos, etc.) y la formación (plan de empresa) han sido financiadas con ahoros propios o familiares, con pequeños préstamos o crowdfunding. Su red comercial es cohesionada (todos se conocen entre sí), presenta muchos lazos fuertes (familiares y amigos) y un nivel de endeudamiento variable: desde empresas familiares que optan a créditos arriesgados, pasando por iniciativas humildes creadas con unos pocos miles de euros, hasta empresas que han sido impulsadas por los programas públicos y privados de emprendimiento.

Por otra parte, como apuntábamos, hallamos un heterogéneo sector de iniciativas orientadas a la venta y a los servicios con un mensaje o valor socio-ambiental añadido. El emprendimiento en este caso suele constituir una alternativa de autoempleo que emerge a raíz de habilidades, destrezas o intereses personales. Este sería el caso de Parla, una empresa de márquetin y (sic) economía del bien común, creada por Andreu, antiguo comercial de una gran empresa de telefonía, con el objetivo de enseñar a comunicar y a hablar en público: "Tengo un don con la comunicación y algo tenía que hacer...". O el caso de L'Escoleta, una escuela de educación libre fundada hace una década por una pareja de maestros desilusionados con el sistema educativo general. O Hydrat, una empresa dedicada a sensibilizar sobre la salud alimenticia ideada por una enfermera a raíz de la enfermedad de su hijo. En otros casos, el valor social o ecológico resulta discutible, como en el caso de una empresa de mensajería en bicicleta para reducir las emisiones de $\mathrm{CO}_{2}$; un circo itinerante creado por una pareja de viajeros desempleados; un taller de estampados de camisetas con mensajes inconformistas; o una correduría de seguros que decide aspectos comerciales de modo asambleario.

A pesar de la diversidad, la mayoría de las pequeñas iniciativas, tanto en el ámbito agroecológico como comercial, comparte similares dificultades y paradojas, como veremos a continuación.

Como hemos indicado antes, los encuestados afirmaron que la principal motivación para crear la iniciativa era medioambiental, personal y, solo en último lugar, económica (entre un 5\% y un 12\%). Susana, por ejemplo, una antigua responsable del departamento $\mathrm{I}+\mathrm{D}$ de una importante marca de productos lácteos, explica que trabajar en aquella compañía no le reportaba satisfacción alguna y que, por ello, decidió establecer su propia cooperativa. Natalia, de L'Ocology, afirma que lo que más 
le motiva es la filosofía de su empresa, no el "querer forrarse". Y Montse, directora de una revista crítica de consumo, buscaba un trabajo "que la llenase", al contrario que su antiguo trabajo en un departamento universitario de informática. Sin embargo, aunque es compatible con lo anterior, en una parte significativa de los casos el emprendimiento se revela como un nicho refugio y una alternativa de autoempleo: entre un $30 \%$ y un $40 \%$ de la muestra partía de una situación de inestabilidad laboral o desempleo y, en el momento en que se realizó la entrevista, más de la mitad afirmó estar pluriempleado en diversos trabajos parciales además de la iniciativa. Por otra parte, la auto-explotación y la precariedad también son habituales en estos contextos, al menos en la fase inicial. Por ejemplo, los trabajadores de la cooperativa ecológica Bastilla se veían obligados a cobrar en negro de manera habitual para subsistir y su propietaria, tras quince años de trabajo rural, afirmaba que estaba "harta de trabajar más de las horas que cobro y que cada vez el precio de la hortaliza ecológica baja más". Otrasnews, una empresa de periodismo alternativo, acumulaba un déficit de $9000 €$ al cabo de un año de su fundación durante el que, afirmaban sus tres socios, no tuvieron vida (solo trabajo). En la Serenidad, una pequeña cooperativa apicultora con una línea comercial y otra ecológica, alternan la recolección de miel con la venta en ferias y la realización de talleres los fines de semana. Y cuando no atienden a sus 100 colmenas se dedican al cultivo. Uno de los socios, pluriempleado porque no llegaba a final de mes a pesar de trabajar seis días a la semana y más de doce horas al día, exclamó: "¡es que todo es trabajo!”.

Incluso en los casos de éxito las condiciones no resultan tampoco totalmente optimistas. Foodchain, por ejemplo, era una empresa liderada por un ingeniero y su hermano economista que deciden asociarse con dos médicos y desarrollar una aplicación informática para detectar intolerancias alimentarias. La idea tiene una gran aceptación por parte de la administración, algunas empresas interesadas en el proyecto e incluso escuelas de negocios como La Salle y Harvard. Pero tras cinco años de intenso trabajo su salario no supera los mil euros mensuales y han tenido que recurrir a otros empleos temporales para subsistir.

Con trabajos tan absorbentes y precarios, la tensión con frecuencia acaba afectando a las relaciones personales. Jaume, de 33 años y dueño de un obrador de pan ecológico, dedica más de 40 horas semanales al trabajo y considera que

(...) si no hubiésemos tenido necesidad, un curro estable que nos gustase, nunca lo hubiésemos montado (...) además, ya puestos, piensas que lo mejor es que sea social y ecológico (...) la cosa nos ha costado varios quebraderos de cabeza y tensiones (...) [es que] es muy difícil mantener la ecuación pareja-socios-padres (...) hemos hecho terapia de pareja (...) y no sabíamos dónde nos estábamos metiendo porque ha costado mucho dinero, esfuerzo y sufrimiento".

Según Andrea, de Bastilla, "a pesar de haberlo dado todo por el trabajo, el tener un hijo me ha hecho darme cuenta de que hay otras cosas importantes en la vida", lo cual le lleva a cuestionar los esfuerzos que supone sacar adelante la cooperativa y se planteaba abandonar esa empresa para buscar otra alternativa laboral más estable.

Pero superada la prueba de fuego de los primeros meses o años, consistente en obtener una mínima rentabilidad, surge un dilema común a todas estas iniciativas: continuar con el modelo de empresa social o variar el modelo de negocio, virar hacia 
formas de gestión más verticales, hacerse autónomos o subcontratar a trabajadores (en ocasiones ya se estaba produciendo un subempleo a mano de obra temporal y sin contrato). El creador de La Falguera, por ejemplo, valoraba reconvertir la cooperativa en un patronato empresarial porque consideraba que prefería "un núcleo pequeño de personas tomando las decisiones". Y Eulàlia, de Campalt, también abogaba por un cambio en la estrategia económica para que "la empresa dé beneficios y salga a flote". Lo mismo se observa en algunas cooperativas de ingeniería y energía renovable, como Rumbol o Crisol. La última fue creada por tres socios ingenieros de 40 años para "cambiar el modelo monopolístico de las empresas de energía españolas", pero pronto pasaron a externalizar el montaje a una subcontrata para mejorar el margen de beneficio. Paradójicamente, por tanto, la subsistencia o expansión pasa por aniquilar la vocación social del proyecto. Y es de este modo como los buenos propósitos del emprendimiento social pueden acabar desvirtuándolo (Ruiz-Herrero, 2015: 21).

Otro contrasentido emana del hecho de que el elevado costo productivo hace que los depositarios finales del producto sean consumidores con elevado nivel adquisitivo, particularmente en el caso de la producción agroecológica. Conferir coherencia en ocasiones implica un gran sacrificio personal:

(...) quiero que los precios sean asequibles para personas normales: yo quiero poder vender a gente como yo, porque si no puedo ni comprarme yo lo que vendo... Sí, la ropa ecológica es cara, los materiales son muy caros...pero yo quiero poder venderle a gente como yo".

Una característica singular de estos emprendedores es que suelen ser conscientes de todas estas contradicciones, es un grupo crítico y reflexivo, en sentido sociológico. Óscar, de Otrasnews, no se considera un emprendedor, puesto que el término "se ha utilizado para impulsar una realidad que no es posible, crear una empresa competitiva de una idea" y, de este modo, reconducir el problema del paro actual en el país. Marta, co-fundadora de Asociación Campalt, realiza una observación clave:

(...) hay empresas a las que se les ha dado mucha difusión y visibilidad en el ámbito del emprendimiento social, pero que en realidad es una imagen algo exagerada (...) y detrás de estas historias hay bastante precariedad e incertidumbre".

Para finalizar, en torno del emprendimiento social se erige una efervescente industria de la consultoría orientada, como nota Fleming, a "transformar el trabajo en una experiencia agradable o, incluso, moral y ética, como el emprendimiento social" (2014: 23). El emprendimiento social se percibe como un nicho de oportunidad tal y como expone Jordi, fundador de la consultoría Steps: "lo social es un nicho de mercado emergente, llamativo y prometedor". No es por ello extraño que la mayoría surja después de 2008 en respuesta a la reconversión del sector y/o como alternativa laboral frente a los recortes públicos y el adelgazamiento de la cartera de clientes.

Las consultorías hacen de conectoras en un mundo poroso en el que, como apunta Xavi, líder de Comencer y antiguo empleado en la administración local, "la articulación estado-agentes es clave, [se requiere] una relación funcional para ambos 
agentes porque el aparato político puede impulsar un tipo de política económica en la que las personas se sientan animadas a formular soluciones a problemas económicos concretos: el autoempleo por ejemplo".

Los casos de Dual y de Multicoop resultan ilustrativos en este sentido. En ambos casos las fundan varios socios que ya contaban con una amplia cartera de proyectos y contactos en el mundo de las fundaciones bancarias, la universidad y la empresa pública y privada. La inversión inicial fue mínima, unos $3000 €$ por socio, y su cartera de clientes incluye proyectos públicos, bancos y escuelas de negocios que les encargan eventos de promoción, talleres y seminarios de emprendimiento social. Estos cursos no difieren demasiado de la formación ofrecida por las escuelas de negocios: "aunque seamos una empresa social, con objetivos sociales, la manera de gestionarla es exactamente igual a como se gestiona una empresa normal", afirma uno de los socios de Dual. En efecto, en los cursos y talleres observados era habitual la participación de profesores universitarios y el empleo de técnicas y estilos de gestión propios de las escuelas de negocios (coaching, etc.). También era habitual encontrar un mensaje final a modo de "baño de realismo" que subrayaba la necesidad de que los proyectos fueran menos sociales y más viables.

Los eventos de promoción suelen seguir un patrón similar. Adoptan llamativos nombres en inglés (Social Startups Meetings, Social Enterpreneurship Workshop, etc.) y consisten en cursos formativos impartidos por consultores a pequeños grupos de personas (unos 20 o 30 alumnos) con un coste de matriculación de entre $400 €$ y $600 €$ por módulo, según lo observado en unos pocos casos. Los cursos suelen ir orientados a potenciales emprendedores alternativos (viajeros, trabajadores del mundo del teatro, vendedores ambulantes y artistas, etc.) para que puedan rentabilizar sus competencias y destrezas mediante iniciativas concretas en forma de carpintería ecológica, reciclaje, cuidados a la tercera edad, risoterapia, o pedagogía medioambiental.

Los cursos van precedidos de ponencias encabezadas por directores de programas de emprendimiento, representantes de la administración pública, emprendedores de referencia, expertos y profesores de escuelas de negocios. En tales actos no es difícil apreciar cierto proselitismo del sector y el ensalzamiento de sus virtudes y potencialidades. Por ejemplo, la directora de un conocido programa de emprendimiento de la administración pública afirmaba que "ahora lo que el país necesita es la creación de nuevas empresas sostenibles, competitivas y comprometidas con la sociedad y con el país". En ese espacio también se enfatizan los nuevos modelos de emprendimiento colectivo y la necesidad de que "los emprendedores identifiquen necesidades, asuman riesgos, generen resultados y sepan gestionar bien los recursos que tienen".

Junto a la pedagogía han florecido una plétora de hubs, espacios de co-working, business incubators, seedspaces y labs, definidos como (sic) centros de actividad de talento para el cambio social, que también constituyen fuentes de ingresos. Para acceder a muchos espacios de co-working social basta con pagar una cuota mensual por la cual se accede a un espacio logístico (un escritorio con conexión a Internet y acceso a algunos servicios) y social (contactos, talleres, otros trabajadores, etc.). Muchos de estos espacios, ubicados en puntos estratégicos de la ciudad, comparten una estética similar: espacios diáfanos y sincréticos (que mezclan minimalismo con estilo vintage, postindustrial y arte pop), inundados de diseños, post-its y notas de automotivación; techos altos de pladur y lampistería a vista, donde ocio y descanso (sofás, pufs) se funden con trabajo (grandes mesas, última tecnología) para promo- 
ver la horizontalidad, la creatividad y la innovación. Similares escenarios se han documentado en la Nueva Economía, la moderna industria del software y los espacios de la clase creativa (Valenzuela, Molina, Lobato et al., 2015). En la práctica, y pese a la ideología comunitaria, en esos espacios uno halla pequeñas empresas que subcontratan un espacio para ahorrarse costos a una comunidad de individuos, entre los que se encuentra Lucía, trabajando frenéticamente con sus laptops y smartphones.

\section{Conclusiones}

En este artículo hemos expuesto el contexto en el que surge el emprendimiento social en Cataluña y hemos descrito sus características centrales. Esto nos ha permitido extraer una serie de conclusiones generales que, posiblemente, sean extensibles a un contexto global.

En primer lugar, el análisis muestra que el emprendimiento social, un sector heredero de la tradición cooperativista, subsume una heterogénea clase "creativa" auto-empleada, desplazada por la crisis y expuesta a una progresiva degradación estructural del trabajo. Aunque la precariedad ha sido siempre una característica del capitalismo, la novedad es que ahora afecta también a los trabajadores cualificados (Gill y Pratt, 2008: 26), que son empujados a formas de emprendimiento y autoempleo desprovistos de amparo institucional.

En segundo lugar, como hemos señalado en otro lugar (Molina, Lubbers, Valenzuela et al., 2018), la definición de empresa social presenta inconsistencias que se revelan en la praxis cotidiana de sus participantes. Por una parte, la definición resulta adscriptiva y prescriptiva simultáneamente: una empresa es social si sus creadores se adscriben a la etiqueta y a la filosofía de una definición emanada del flujo sostenido de ideas de arriba hacia abajo (Hervieux, Gedajlovic y Turcotte, 2010). Esto explicaría el conjunto heterogéneo, y a menudo contradictorio, de iniciativas halladas en este sector. De hecho, la definición de empresa social es tautológica o redundante (Santos, 2012; Doherty, Haugh y Lyon, 2014; Lall, 2017), posiblemente por su naturaleza híbrida, que trata de casar dos principios auto-excluyentes (solidaridad y justicia versus mercado capitalista). Y esta indefinición se traduce en la dificultad para mantener el compromiso entre beneficio económico y bien común. Además, si bien el desempeño económico es relativamente sencillo de mostrar (por el nivel de ingresos, ventas, capitalización, crecimiento, número de trabajadores, etc.) el impacto social no es solo difícil de medir sino también de definir (Manetti, 2012; Young y Lecy, 2014). Por ello se advierte una tendencia a equiparar el impacto social con la productividad (Arvidson y Lyon, 2014). Lall (2017), tras analizar una base de datos de 1864 empresas sociales emergentes, confirma que la adopción de prácticas de SPM (social performance measurement) responde a una creciente racionalización económica del sector. En realidad, los emprendedores sociales usan los mismos recursos que las empresas comerciales (incubadoras, coaching, planes de negocio, márquetin, acceso crédito, etc.) y, como hemos apuntado, los cursos y eventos de promoción del emprendimiento social son a menudo vehículos para inocular criterios de viabilidad, eficiencia y racionalidad propios del sector comercial (Austin, Stevenson y Wei-Skillern, 2006; Meyskens, Robb-Post, Stamp et al., 2010). Ese camino parece conducir inexorablemente a desvirtuar el carácter social, solidario y alternativo del emprendimiento social. 
En tercer lugar, el emprendimiento social encarna la culminación de diversos desarrollos del capitalismo avanzado: la industria cognitiva, el movimiento del capital humano y el emprendimiento. Este hecho sugiere no solo una progresiva expansión de la ideología neoliberal al resto de las esferas sociales, sino también una forma de extracción de valor a coste cero para el Estado y la industria financiera: el empresariado social genera trabajo; ofrece respuestas innovadoras y creativas (medios de diferenciación y competitividad en los mercados); asume todo el riesgo y los costes; ahorra el gasto a un Estado que ha delegado (externalizado) sus funciones (inserción de personas vulnerables, resolución de problemas sociales...) y genera valor que trata de reabsorber el capital convirtiendo las soluciones sociales en mercancías (en forma de servicios facturables, derechos, patentes, prototipos, ideas, etc.). En este sentido el emprendimiento social es un artefacto estratégico para legitimar la "privatización del interés público" (Kamat, 2004) ${ }^{11}$. Por esa razón, alrededor de este emprendimiento se erige una creciente industria compuesta por consultores, espacios de co-working, eventos, cursos de formación y venta de servicios disputados por los distintos actores (actores financieros, administración pública, empresarios comerciales, etc.).

Finalmente, y de acuerdo con la literatura consultada, el emprendimiento social no es un fenómeno específico, sino que sugiere una reestructuración global del trabajo (de individuos carentes de apoyo institucional), basada en la lógica del emprendimiento y la creciente externalización de las funciones del Estado.

\section{Referencias bibliográficas}

Adkins, Lisa (2002). Revisions: Gender and Sexuality in Late Modernity. Buckingham and Philadelphia: Pen University Press.

Arvidson, Malin; Lyon, Fergus (2014). "Social Impact Measurement and Non-profit Organisations: Compliance, Resistance, and Promotion". VOLUNTAS: International Journal of Voluntary and Nonprofit Organizations, 25 (4): 869-886.

Austin, James; Howard, Stevenson; Wei-Skillern, Janes (2006). "Social and Commercial Entrepreneurship: Same, Different, or Both?" Entrepreneurship. Theory and Practice, 30 (1): $1-22$.

Bayley, Geoff(2014). “Accumulation by dispossession. Acritical assessment”. ISR. International Socialist Review, 95 [https://isreview.org/issue/95/accumulation-dispossession].

Beck, Ulrich; Giddens, Anthony; Lash, Scott (1994). Reflexive Modernization. Politics, Tradition and Aesthetics in the Modern Social Order. Stanford: Stanford University Press.

Beck, Ulrich (1992). Risk Society: Towards a New Modernity. London: SAGE.

Beck, Ulrich; Beck-Gernsheim Elisabeth (2002). Individualization: institutionalized individualism and its social and political consequences. London: SAGE.

Beck, Ulrich; Bonss, Wolfgang; Lau, Christoph (2003). "The theory of reflexive modernization: problematic, hypotheses and research". Theory, Culture \& Society 20 (2): 1-33.

Benner, Chris (2002). Work in the New Economy: Flexible Labor Markets in Silicon Valley. Malden: Blackwell.

11 Fleming considera que el Capitalismo está reinventando su propia versión de comunidad (2014: 17) mediante una portentosa propaganda que pone en circulación atractivos conceptos, como economía colaborativa, inteligencia colectiva, co-diseño, co-trabajo o emprendimiento social. 
Bornstein, David; Davis, Susan (2010). Social Entrepreneurship. What Everyone Needs To Know. New York: Cambridge University Press.

Booth, William J. (1994). "On the idea of the Moral Economy". The American Political Science Review, 88(3): 653-667.

Clifton, Nick (2008). "The "creative class" in the UK: an initial analysis". Geografiska Annaler B, 90 (1): 63-82.

Conde-Ruiz, Jose Ignacio; Díaz, Manuel; Marín, Carlos; et al. (2016). Sanidad, Educación y Protección Social: Recortes Durante la Crisis. FEDEA, Observatorio Fiscal y Financiero de las Comunidades Autónomas. Madrid.

Dacin, Peter A.; Dacin, M. Tina; Matear, Margaret (2010). "Social Entrepreneurship: Why We Don't Need a New Theory and How We Move Forward From Here". The Academy of Management Perspectives, 24(3):37-57.

Dacin, M. Tina; Dacin, Peter A.; Tracey, Paul (2011). "Social entrepreneurship: A critique and future direction". Organization Science, 22 (5):1203-1213.

De Witte, Hans (1999). Job Insecurity and Psychological Well-Being: Review of the Literature and Exploration of Some Unresolved Theory. Oxford, UK: Oxford University Press.

Del Pino, Eloísa (2013). "The Spanish Welfare State from Zapatero to Rajoy: Recalibration to Retrenchment", en B. N. Field \& A. Botti (Eds.). Politics and Society in Contemporary Spain: From Zapatero to Rajoy. New York: Palgrave Macmillan, 197-216.

Díaz Bretones, Francisco (2000). "Desempleo y cooperativismo". CIRIEC-España, Revista de Economía Pública, Social y Cooperativa, 35: 161-176.

Dilly, Barbara J. (2006). "Volunteer Labor: “Adding Value” to Local Culture”, in Durrenberger, E.P., and Judith Martí (eds.) (2006) Labor in Cross-Cultural Perspective. Lanham, NY, London Oxford: Altamira Press, Rowman and Littlefield Pubs.

Doherty, Bob; Haugh, Hellen; Lyon, Fergus (2014). "Social Enterprises as Hybrid Organizations: A Review and Research Agenda". International Journal of Management Reviews, 16 (4): 417-436.

Driver, Michaela (2012). “An Interview With Michael Porter: Social Entrepreneurship and the Transformation of Capitalism". Academy of Management Learning \& Education, 11(3): 421-431.

Drucker, Peter (1968). The age of discontinuity. Guidelines for our changing society. New York: Harper and Row.

EURICSE (2013). Social economy and social entrepreneurship. Luxembourg: Social Europe guide.

Ferguson, James (2010). "The Uses of Neoliberalism”. Antipode 41:166-84.

Fleming, Peter (2014). Resisting work. The cooperatization of life and its discontents. Philadelphia: Temple University Press.

Florida, Richard (2010). La clase creativa. La transformación de la cultura del trabajo y el ocio en el siglo XXI. Madrid: Paidós empresa.

Foucault, Michel (2008). The Birth of Biopolitics. Lectures at the Collège de France, 19781979. London: Palgrave.

Fraser, Jill (2002). White Collar Sweatshop: The Deterioration of Work and Its Rewards in Corporate America. Nueva York: W. W. Norton.

Freeman, Carla (2007). “The 'Reputation' of neoliberalism”. American Ethnologist 34 (2): 252-67.

Gill, Rosalind; Pratt, Andy C. (2008). "In the social factory? Immaterial labour, precariousness and cultural work". Theory, Culture \& Society, 25(7-8): 1-30. 
Hardt, Michael: Negri, Antonio (2000). Empire. Cambridge, Massachusetts, London: Harvard University Press.

Hart, Keith; Laville, Jean-Louis; Cattani, Antonio David (2010). Human Economy: A Citizen's Guide. Polity Press.

Hart, Keith (Ed.) (2015). Economy for and Against Democracy. Col. The Human Economy, vol. 2. NY, Oxford: Berghahn.

Harvey, David (2007a). A brief History of Neoliberalism. Oxford: OUP.

Hervieux, Chantal; Gedajlovic, Eric; Turcotte, Marie-France (2010). "The Legitimization of Social Entrepreneurship". Journal of Enterprising Communities: People and Places in the Global Economy, 4(1): 37-67.

Hilgers, Mathieu (2012). "The Historicity of the Neoliberal State. Social Anthropology, 20 (1): 80-94.

Hunt, Lesley (2006). "Living with Uncertainty: Estrangement, Alienation and Agency At Work". Sociological Association of Aotearoa, New Zealand, 2003, Agribusiness and Economics Research Unit (AERU), Lincoln University.

Inglehart, Ronald (1977). The Silent Revolution. Princeton: Princeton University Press.

Inglehart, Ronald (2009). El cambio cultural en las sociedades industriales avanzadas [Culture Shift. In Advanced Industrial Society, 1990]. Madrid: CIS.

Jorrín, Javier G. (2017). "El Banco de España cifra en 60.600 millones las pérdidas por el rescate a la banca". El Confidencial. [Consultado el 14/08/2018, disponible en https:// www.elconfidencial.com/economia/2017-06-16/rescate-bancario-coste-perdido-bancoespana-bde_1400328].

Kalleberg, Arne L. (2009). "Precarious Work, Insecure Workers : in Transition Employment Relations". American Sociological Review, 74(1): 1-22.

Kamat, Sangeeta (2004). "The Privatization of Public Interest: Theorizing NGO Discourse in a Neoliberal Era”. Review of International Political Economy, 11(1):155-176.

Koslowski, Peter (2008). Principles of Ethical Economy, Issues in Business Ethics. Springer.

Kunda, Gideon (1992). Engineering culture. Control and commitment in a high-tech corporation. Philadelphia: Temple University Press.

Lang, Peter (1994). LETS Work: Rebuilding the Local Economy. Bristol: Grover Books.

Lall, Saurabh (2017). "Measuring to Improve Versus Measuring to Prove: Understanding the Adoption of Social Performance Measurement Practices in Nascent Social Enterprises". VOLUNTAS: International Journal of Voluntary and Nonprofit Organizations, 28 (6): 2633-2657.

Linton, Michael (1994). The LETS System Design Manual. Landsman Community Services Paper 1.3 Version 1.3. Birgingham: Birmingham Co-operative.

Littlewood, David; Holt, Diane (2018). "Social Entrepreneurship in South Africa: Exploring the Influence of Environment”. Business \& Society, 57(3): 525-561.

Lovink, Geert (2007). “Organic Intellectual Work: Interview with Andrew Ross" [Consultado el 11/07/2017, disponible en http://networkcultures.org/wpmu/geert/interviewwithandrew-ross]

McRobbie, Angela (2010). "Clubs to companies: notes on the decline of political culture in speeded up creative worlds". Cultural Studies 16(4): 516-531.

Manetti, George (2012). "The Role of Blended Value Accounting in the Evaluation of SocioEconomic Impact of Social Enterprises". VOLUNTAS: International Journal of Voluntary and Nonprofit Organizations, 25(2): 443-464.

Martín Belmonte, Susana (2011). Nada está perdido: Un sistema monetario y financiero alternativo y sano. Barcelona: Icaria Editorial. 
Mason, Paul (2015). Postcapitalism: a guide to our future. UK: Penguin.

Mendell, Marguerite (2005). Reclaiming Democracy: The Social Justice and the Political Economy of Gregory Baum and Kari Polanyi Levitt. Quebec: McGill-Queen's University Press.

Meyskens, Moriah; Robb-Post, Calleen; Stamp, Jeffrey A.; et al. (2010). "Social Ventures from a Resource-Based Perspective: An Exploratory Study Assessing Global Ashoka Fellows". Entrepreneurship. Theory and Practice Theory and Practice, 34(4): 661-680.

Lobato, Marta; Molina, Jose Luis; Valenzuela-Garcia, Hugo (2018a) "Cross-cutting Precariousness: Values, Work and Inequality in Post-2008 Spain”. Urbanities 8 (1): 65 79.

Lobato, Marta; Molina, Jose Luis; Valenzuela-Garcia, Hugo (2018b) "Individualization, Inequality and Labour: A Qualitative approach". Dialectical Anthropology, 42(3): 27729.

Molina, José Luis; Lubbers, Miranda J.; Valenzuela, Hugo; et al. (2018) "The Cowl Does Make The Monk": Understanding the Emergence of Social Entrepreneurship in Times of Downturn". VOLUNTAS, 29 (4), 725-739.

Montero, María Teresa; Arcenegui Rodrigo, José Antonio; Martín Lozano, José Manuel; et al. (2015). "El comportamiento social en las cajas de ahorros españolas durante la crisis". Prisma Social: Revista de Ciencias Sociales 14: 516-544.

Moon, Chul Woo; Koh, You Sang (2015). "Compassion, Pro-Social motivation, and Social Entrepreneurship: An Empirical Investigation", Proceedings of the Third Asia-Pacific Conference on Global Business, Economics, Finance and Banking (AP15Singapore Conference) ISBN:978-1-63415-751-317-19 July 2015 Paper ID: S580

Moulaert, Frank; Ailenei, Oana (2005). "Social economy, third sector and solidarity relations: A conceptual synthesis from history to present". Urban Studies, 42(11): 2037-2054.

Mount, Ingl (2014). "España vive 'boom' de trabajo informal”, CNN Expansión, 17 feb. [Recurso online consultado el 12/2/2015, disponibe en: https://expansion.mx/ economia/2014/02/14/economia-informal-de-espana-en-auge ]

Overell, Stephen (2008). Inwardness: The Rise of Meaningful Work. Provocation Series, The Work Foundation, 4(2), 48. Recurso online: http://www.theworkfoundation.com/ DownloadPublication/Report/32_32_inwardness_fi

Pettifor, Ann (2015). "La corrupción del sistema financiero es una tragedia". Revista Contexto 108, 15/03/2017. Recurso online consultado el 14/08/2018: http:/ctxt.es/es/20170315/ Firmas/11680/banco-de-espa $\%$ C3\%B1a-malversacion-rodrigo-rato-bankia-sistemafinanciero-ann-pettifor.htm

Pfeilstetter, Richard (2011). "The entrepreneur. A critical reflection on the current uses and meanings of a concept". Gazeta de Antropologia, 27(1):1-11.

Polanyi, Karl (1977). El sustento del hombre. Madrid: Capitán Swing, 2009.

Prentice, Rebecca (2015). Thiefing a Chance. Factory Work, Illicit Labor, and Neoliberal Subjectivities in Trinidad. US: Universty Press of Colorado.

Reventós, Josep (1960). El movimiento cooperativo en España. Barcelona: Ariel.

Romani, Michela (2014). "La banca cerró 2013 con unos 9.000 millones de euros de beneficio". Expansión, 19 ene. [Consultado el 12/06/2017, disponible en http://www. expansion.com/2014/01/17/empresas/banca/1389963101.html]

Rose, Nikolas (1999). Powers of Freedom: Reframing Political Thought. Cambridge: Cambridge University Press.

Ross, Andrew (2003). No-collar: the human workplace and its hidden costs. Nwe York: Basic Books. 
Rowan, Jaron (2010). Emprendizajes en cultura. Discursos, instituciones y contradicciones de la empresarialidad cultural. Madrid: Traficantes de sueños.

Ruiz-Herrero, Jesús Antonio (2015). Capitalismo cognitivo: sus nuevos espacios, técnicas de control y producción, y sus clases profesionales: dos estudios de caso. Tesis Doctoral. Departamento de Sociología. Universidad Complutense Madrid. Consulta on-line: https:// www.educacion.gob.es/teseo/mostrarRef.do?ref=357174 [consultado el 29/08/2018].

Sahlins, Marshall (1972). Stone Age Economics. Nueva York: Routledge.

Santos, Felipe M. (2012). "A Positive Theory of Social Entrepreneurship". Journal of Business Ethics, 111(3): 335-351.

Scace, Richard (2002). Living in the Corporate Zoo. Life and Work in 2010. UK: Capostone Publishing Limited.

Schumpeter, Joseph A. (1942). Capitalismo, socialismo y democracia. Barcelona: Ediciones Orbis, 1983.

Scott, James (1976). The Moral Economy of the Peasant. Yale: Yale Universty Press.

Sennet, Richard (1992). The Fall of Public Man.New York: W. W. Norton \& Company.

Sennet, Richard (2000). The Corrosion of Character: The Personal Consequences of Work in the New Capitalism. New York: W. W. Norton \& Company.

Terranova, Tiziana (2000). "Free Labor: Producing Culture for the Digital Economy". Social Text, 63(18, 2): 33-58.

Thompson, Edward. P. (1971). "The Moral Economy of the English Crowd in the 18th Century". Past \& Present, 50: 76-136.

Valenzuela-Garcia, Hugo; Fuentes, Sandrine (2019). "A Crossroads for Social Entrepreneurship: ethics versus profit in Spain". Open Journal of Business and Management, 7(2):848-860.

Valenzuela-Garcia, Hugo; Reygadas, Luis; Cruces, Francisco (2015). "Mi trabajo es mi vida. La incrustación de los mundos de la vida y del trabajo en empresas españolas". Revista Española de Investigaciones Sociológicas, 150: 191-210.

Valenzuela, Hugo; Molina, Jose Luis; Lobato, Marta; et al. (2015). "Empresas sociales en Cataluña ¿Cambio de paradigma o estrategia de clase media?”. Otra Economía: revista latinoamericana de economía social y solidaria, 9 (17): 177-186.

Wacquant, Loïc (2009). Punishing the Poor. The Neoliberal Government of Social Insecurity. Duke University Press.

Waters, Cara (2017). "The lives and lies of social entrepreneurs". The Sydney Morning Herald. Recurso on-line consultado el 15/11/2018: https://www.smh.com.au/business/ small-business/the-lifes-and-lies-of-social-entrepreneurs-20170210-guaghc.html]

Young, Donald R.; Lecy, John D. (2014). "Defining the Universe of Social Enterprise: Competing Metaphors". Voluntas, 25(5), 1307-1332.

Zak, Paul J. (Ed.) (2008). Moral Markets. The Critical Role of Values in the Economy. Princeton: Princeton University Press. 